Hexel, Erhard:

On short cycles through prescribed vertices of a polyhedral graph

URN: $\quad$ urn:nbn:de:gbv:ilm1-2020200129

\begin{tabular}{|c|c|}
\hline Original published in: & $\begin{array}{l}\text { Discussiones mathematicae. Graph theory / Uniwersytet Zielonogórski, } \\
\text { Wydział Matematyki, Informatyki i Ekonometrii. - Warsaw : De Gruyter } \\
\text { Open. - } 25 \text { (2005), 3, p. 419-426. }\end{array}$ \\
\hline Original published: & 2005 \\
\hline ISSN: & 2083-5892 \\
\hline DOI: & 10.7151/dmgt.1293 \\
\hline \multirow[t]{2}{*}{ [Visited: } & 2020-01-17] \\
\hline & $\begin{array}{l}\text { This work is licensed under a Creative Commons Attribution- } \\
\text { NonCommercial-NoDerivatives } 3.0 \text { Unported license. } \\
\text { To view a copy of this license, visit } \\
\text { http://creativecommons.org/licenses/BY-NC-ND/3.0/ }\end{array}$ \\
\hline
\end{tabular}


Discussiones Mathematicae

Graph Theory 25 (2005) 419-426

\title{
ON SHORT CYCLES THROUGH PRESCRIBED VERTICES OF A POLYHEDRAL GRAPH
}

\author{
ERHARD HEXEL \\ Department of Mathematics \\ Technische Universität Ilmenau \\ Postfach 0565, D-98684 Ilmenau, Germany
}

\begin{abstract}
Guaranteed upper bounds on the length of a shortest cycle through $k \leq 5$ prescribed vertices of a polyhedral graph or plane triangulation are proved.
\end{abstract}

Keywords: polyhedral graph, triangulation, short cycle, prescribed vertices.

2000 Mathematics Subject Classification: 05C38.

\section{Introduction and Results}

G.A. Dirac [2] proved that for a given integer $c \geq 2$ any $k(1 \leq k \leq c)$ prescribed vertices of a $c$-connected graph belong to a common cycle. However, the complete bipartite graph $K_{c, c+1}$ shows that this is not true for $c+1$ prescribed vertices. In [3] we investigated the length of short cycles through $k$ prescribed vertices with $1 \leq k \leq \min \{c, 3\}$ in a $c$-connected graph $G$. From A.K. Kelmans and M.V. Lomonosov [6] we know that any five vertices of a polyhedral graph (that is a planar and 3-connected graph) belong to a common cycle which is best possible.

For given integers $k, l$ with $1 \leq k \leq 5,3 \leq l$ and $k \leq l$ let $n_{k}(l)$ denote the minimum number $n$ such that there exists a polyhedral graph $G$ of order $n$ having a subset of $k$ vertices with the property that the length of every cycle containing those $k$ vertices is at least $l$. In [3] we proved

(i) $n_{1}(l)=3 l-5$ for $l \geq 3$, 
(ii) $n_{2}(l)=\left\lfloor\frac{3 l-1}{2}\right\rfloor$ for $l \geq 3$,

(iii) $n_{3}(l)=\left\lfloor\frac{3 l-1}{2}\right\rfloor$ for $l \geq 5$,

and the following results which will be proven here is a continuation of the investigation [3] of short cycles through prescribed vertices for a polyhedral graph.

\section{Theorem 1.}

$$
n_{4}(l)=\left\{\begin{array}{cl}
l & \text { if } l \in\{4,8\}, \\
l+1 & \text { if } l \in\{5,6,7,9,10\} \\
l+2 & \text { if } l \in\{11,12\} \\
\left\lceil\frac{4 l-5}{3}\right\rceil & \text { if } l \geq 13 .
\end{array}\right.
$$

\section{Theorem 2.}

$$
n_{5}(l)=\left\{\begin{array}{cll}
l & \text { if } l=5 & \text { or } l \geq 8 \\
l+1 & \text { if } l=6 & \text { or }
\end{array}\right.
$$

For integers $k, l$ with $2 \leq k \leq 5,3 \leq l$ and $k \leq l$ denote by $t_{k}(l)$ the minimum number $n$ such that there exists a plane triangulation $T$ of order $n$ with certain $k$ vertices such that the length of every cycle containing them is at least $l$. Then we have $n_{k}(l) \leq t_{k}(l)$ since every plane triangulation is 3 -connected and thus a polyhedral graph. Notice that even $n_{k}(l)=t_{k}(l)$ holds in every considered case. If, namely, $G$ is any one of the here or in [3], respectively, constructed graphs to prove an upper bound for $n_{k}(l)$ with certain $k$ and $l$, then we were able to construct a plane triangulation $T$ from $G$ by adding edges only such that the length of a shortest cycle containing the prescribed $k$ vertices is at least $l$.

\section{Proofs}

For terminology and notation not defined here we refer to [5]. Let $G$ be a graph and $A, B \subseteq V(G)$. A path $P$ of $G$ with one end-vertex in $A$ and $B$, respectively, and with $|V(P) \cap A|=|V(P) \cap B|=1$ is called an $A$-B-path. If $A$ or $B$ consists of a single vertex $x$ we write $x$ instead of $\{x\}$. We use $[x, y]$ to denote an $x$-y-path and, moreover, $[x, y)$ or $(x, y)$ to denote the segments obtained from $[x, y]$ by removing $y$ or both end-vertices, respectively. A path 
system is a set of internally disjoint paths. For a path system $\mathcal{P}$ let $[\mathcal{P}]$ and $E V(\mathcal{P})$ denote the union of all paths and the set of all end-vertices of paths of $\mathcal{P}$, respectively. For some $a \in V(G)$ and $B \subseteq V(G) \backslash\{a\}$ a path system $\mathcal{P}$ of $a$-B-paths is called an $a-B$-fan if $P \cap Q=\{a\}$ for different $P, Q \in \mathcal{P}$.

We need the following lemma which is proved in [3] in more general form.

Lemma 1. Let $G$ be a c-connected graph with $a \in V(G), B \subseteq V(G) \backslash\{a\}$ and a path system $\mathcal{P}$ of $c-1$ a-B-paths. Let $B^{\prime}=B \backslash E V(\mathcal{P})$ if this is not empty, and $B^{\prime}$ be an arbitrary nonempty subset of $B$ otherwise. Then there is a vertex $b \in B^{\prime}$ and a path system $\mathcal{Q}$ of $c$ a-B-paths such that $E V(\mathcal{Q})=E V(\mathcal{P}) \cup\{b\}$, all vertices of $B \backslash\{b\}$ are end-vertices of as many paths of $\mathcal{P}$ as of $\mathcal{Q}$, and $\mathcal{Q}$ has one more path with end-vertex $b$ than does $\mathcal{P}$.

We define five polyhedral graphs containing the vertices of a prescribed 4element set $X$ as follows. Let $F_{1}$ be the complete graph $K_{4}$ on $X$. Let $F_{2}$ denote the graph which is obtained from a 4-cycle $C$ on $X$ by connecting an additional vertex $a \notin X$ with all vertices of $C$. Let $F_{3}$ denote the graph which results from $C$ and two adjacent vertices $a, b \notin X$ by connecting two adjacent vertices of $C$ with $a$ and the remaining two vertices of $C$ with $b$. The graph $F_{4}$ is obtained if two non-adjacent vertices $a, b \notin X$ are connected with three vertices of a 4-path $P$ on $X$, respectively, such that every vertex of $X$ becomes degree 3 . Eventually, let $F_{5}$ denote the cube graph containing the vertices of $X$ such that no two vertices of $X$ are adjacent.

Lemma 2. Every polyhedral graph $G$ with $X=\left\{x_{1}, x_{2}, x_{3}, x_{4}\right\} \subseteq V(G)$ has a subgraph $H$ which is a subdivision of some $F_{i}$ with $1 \leq i \leq 5$.

Proof of Lemma 2. Lemma 1 implies that $G$ has an $x_{1}-x_{2}$-path system $\left\{P_{1}, P_{2}, P_{3}\right\}$ which contains $x_{3}$ by planarity of $G$, i.e., we may assume that $x_{3} \in V\left(P_{1}\right)$. Moreover, Lemma 1 yields an $x_{3}-V\left(P_{2} \cup P_{3}\right)$-fan $\mathcal{Q}=\left\{\left[x_{1}, x_{3}\right],\left[x_{2}, x_{3}\right],\left[a, x_{3}\right]\right\}$, where we may assume that $a \in V\left(P_{2}\right)$. Thus, $G$ has a path system $\mathcal{P}=\left\{\left[x_{1}, x_{2}\right],\left[x_{1}, x_{3}\right],\left[x_{2}, x_{3}\right],\left[a, x_{1}\right],\left[a, x_{2}\right],\left[a, x_{3}\right]\right\}$.

Suppose first, that $x_{4}$ is contained in $[\mathcal{P}]$. Considering symmetries we have to examine three different cases.

Case 1. $x_{4}=a$.

Then $[\mathcal{P}]$ is a subdivision of $F_{1}$. 
Case 2. $x_{4} \in\left(x_{1}, x_{2}\right)$.

By Lemma 1 there is an $x_{4}-V\left([\mathcal{P}] \backslash\left(x_{1}, x_{2}\right)\right)$-fan $\mathcal{Q}=\left\{\left[x_{1}, x_{4}\right],\left[x_{2}, x_{4}\right],\left[b, x_{4}\right]\right\}$ where $b \in V\left([\mathcal{P}] \backslash\left(x_{1}, x_{2}\right)\right)$. Let $H$ denote the subgraph $[\mathcal{P} \cup \mathcal{Q}] \backslash\left(x_{1}, x_{2}\right)$ of $G$, then by symmetries there are following subcases. If $b=x_{3}$ or $b=a$ then $H$ is a subdivision of $F_{1}$ or $F_{2}$, respectively. If $b \in\left(x_{1}, x_{3}\right)$ or $b \in\left(a, x_{1}\right)$ then $H$ is a subdivision of $F_{4}$ or $F_{3}$, respectively.

Case 3. $x_{4} \in\left(a, x_{1}\right)$.

Applying Lemma 1 again there is an $x_{4}-V\left([\mathcal{P}] \backslash\left(a, x_{1}\right)\right)$-fan $\mathcal{Q}=\left\{\left[x_{1}, x_{4}\right],[a\right.$, $\left.\left.x_{4}\right],\left[b, x_{4}\right]\right\}$ where $b \in V\left([\mathcal{P}] \backslash\left(a, x_{1}\right)\right)$. Let $H$ denote the subgraph $[\mathcal{P} \cup \mathcal{Q}] \backslash$ $\left(a, x_{1}\right)$ of $G$. Considering symmetries we have: If $b \in\left(x_{1}, x_{2}\right)$ or $b \in\left[x_{2}, a\right)$ then $H$ is a subdivision of $F_{4}$ or $F_{1}$, respectively.

Suppose now, that $x_{4}$ is not contained in $[\mathcal{P}]$ and in any other such path system of $G$. Applying Lemma 1 we obtain an $x_{4^{-}} V([\mathcal{P}])$-fan $\mathcal{Q}=\left\{\left[b, x_{4}\right]\right.$, $\left.\left[c, x_{4}\right],\left[d, x_{4}\right]\right\}$ such that each path of $\mathcal{P}$ contains at most one vertex of $E V(\mathcal{Q})$ and that at most one path of $\mathcal{P}$ with end vertex a contains a vertex of $E V(\mathcal{Q})$. Thereby and since $G$ is planar we may assume that $b \in\left(x_{1}, x_{2}\right), c \in\left(x_{2}, x_{3}\right)$ and $d \in\left(x_{1}, x_{3}\right)$ which implies that $[\mathcal{P} \cup \mathcal{Q}]$ is a subdivision of $F_{5}$.

Figure 1 contains further three polyhedral graphs which contain the vertices of $X=\left\{x_{1}, x_{2}, x_{3}, x_{4}\right\}$ and which are needed to prove Theorem 1 .

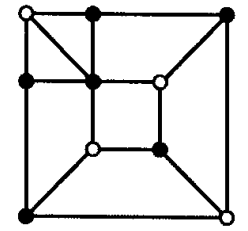

$F_{6}$

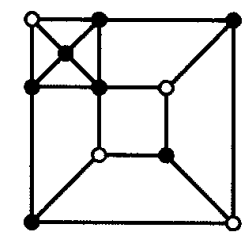

$F_{7}$

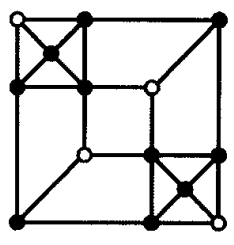

$F_{8}$

Figure 1

Proof of Theorem 1. For $l=6,7,11$ and $l \geq 13$ connect a vertex $a$ with each vertex of a 4 -cycle $C=x_{1} x_{2} x_{3} x_{4} x_{1}$. Put $\alpha=\left\lfloor\frac{l-5}{3}\right\rfloor$ and suppose $l \equiv r(\bmod 3)$ where $r \in\{0,1,2\}$. Subdivide every edge $e$ of $C$ with respect to $r$ by the number of new vertices given in Table 1 . Connect every new vertex with $a$ and denote the so constructed polyhedral graph by $G$. 
Table 1

\begin{tabular}{c|cccc}
$r \backslash e$ & $x_{1} x_{2}$ & $x_{2} x_{3}$ & $x_{3} x_{4}$ & $x_{4} x_{1}$ \\
\hline 0 & $\alpha+1$ & $\alpha+1$ & $\alpha$ & $\alpha$ \\
1 & $\alpha+1$ & $\alpha+1$ & $\alpha+1$ & $\alpha$ \\
2 & $\alpha$ & $\alpha$ & $\alpha$ & $\alpha$
\end{tabular}

A simple calculation shows that the length of a shortest cycle in $G$ containing $X=\left\{x_{1}, x_{2}, x_{3}, x_{4}\right\}$ is $l$ and that the order of $G$ is $\left\lceil\frac{4 l-5}{3}\right\rceil$, in every case.

For $l=4,5,8,9,10,12$ let $G$ be $F_{1}, F_{4}, F_{5}, F_{6}, F_{7}, F_{8}$, respectively, with $X \subseteq V(G)$. In these special cases it is not hard to see that the length of a shortest cycle of $G$ containing $X$ is $l$. That together with $n_{4}(l) \leq|G|$ completes the proof of the upper bound.

Suppose, now, that $G$ is a polyhedral graph of order $n$ with a 4-element subset $X=\left\{x_{1}, x_{2}, x_{3}, x_{4}\right\}$ of $V(G)$ such that the length of a shortest cycle containing $X$ is at least $l$. Because of Lemma 2 it is sufficient to estimate for $i=1, \ldots, 5$ the order of a subgraph $H$ of $G$ which is a subdivision of $F_{i}$ with $X \subseteq V\left(F_{i}\right)$ and to deduce a lower bound for $n_{4}(l)$.

$i=1$ : $H$ has three different cycles $C_{1}, C_{2}, C_{3}$ passing each vertex of $F_{1}$. Every vertex of $V(H) \backslash V\left(F_{1}\right)$ occurs in precisely two of these three cycles. Thus, $2|H|+4 \geq\left|C_{1}\right|+\left|C_{2}\right|+\left|C_{3}\right| \geq 3 l$ and, consequently, $|H| \geq\left\lceil\frac{3 l-4}{2}\right\rceil$.

$i=2: H$ has four cycles $C_{1}, \ldots, C_{4}$ containing all vertices of $F_{2}$ and one cycle $C_{5}$ containing $X$ but no other vertex of $F_{2}$. Every vertex of $V(H) \backslash$ $V\left(F_{2}\right) \backslash V\left(C_{5}\right)$ occurs in precisely two and every vertex of $V\left(C_{5}\right) \backslash V\left(F_{2}\right)$ in precisely three of the cycles $C_{1}, \ldots, C_{4}$. Thus, $2|H|+\left|C_{5}\right|+4 \cdot 1+2 \geq$ $\left|C_{1}\right|+\ldots+\left|C_{4}\right| \geq 4 l$ and, thereby, $2|H|+\left|C_{5}\right|+6 \geq 4 l$. From $\left|C_{5}\right| \leq|H|-1$ we further obtain $|H| \geq\left\lceil\frac{4 l-5}{3}\right\rceil$.

$i=3,4: H$ has three different cycles $C_{1}, C_{2}, C_{3}$ passing each vertex of $F_{i}$. Every vertex of $V(H) \backslash V\left(F_{i}\right)$ occurs in precisely two of these three cycles. Thus, $2|H|+6 \geq\left|C_{1}\right|+\left|C_{2}\right|+\left|C_{3}\right| \geq 3 l$ and, consequently, $|H| \geq\left\lceil\frac{3 l-6}{2}\right\rceil$.

$i=5$ : $H$ has six different cycles $C_{1}, \ldots, C_{6}$ passing each vertex of $F_{5}$. Every vertex of $V(H) \backslash V\left(F_{5}\right)$ occurs in precisely four of these six cycles. Thus, $4|H|+2 \cdot 8 \geq\left|C_{1}\right|+\ldots+\left|C_{6}\right| \geq 6 l$ and, consequently, $|H| \geq\left\lceil\frac{3 l-8}{2}\right\rceil$. Because of $|G| \geq \min \left\{\left|H_{i}\right|: 1 \leq i \leq 5\right\}$ and $|G| \geq l$ we obtain 


$$
n_{4}(l) \geq\left\{\begin{array}{cl}
l & \text { if } l \in\{4,5,6,8\}, \\
l+1 & \text { if } l \in\{7,9,10\}, \\
l+2 & \text { if } l \in\{11,12\}, \\
\left\lceil\frac{4 l-5}{3}\right\rceil & \text { if } l \geq 13 .
\end{array}\right.
$$

In the special cases $l=5,6$ one can observe that since $G$ has a subgraph $H$ which is a subdivision of $F_{i}$ for some $i \in\{1, \ldots, 5\}$ the order of $G$ can not be smaller than 6 or 7 , respectively. That proves the lower bound.

Proof of Theorem 2. For $l=5,6,7,8,9$ let $G_{l}$ be the polyhedral graphs with $X=\left\{x_{1}, \ldots, x_{5}\right\} \subseteq V\left(G_{l}\right)$ given in Figure 2 .

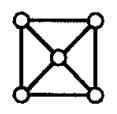

$G_{5}$

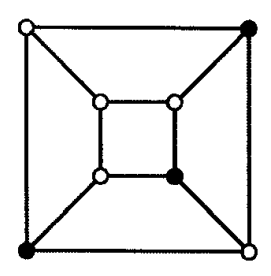

$G_{8}$

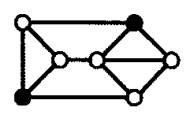

$G_{6}$

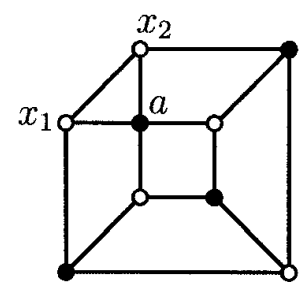

$G_{9}$

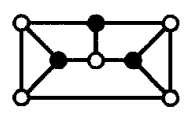

$G_{7}$

$G_{9}$

Figure 2

For $l>9$ let $G_{l}$ be the polyhedral graph which results from $G_{9}$ by subdividing $x_{1} x_{2}$ by $l-9$ new vertices and connecting each of them with $a \notin X$. Notice that $\left|G_{l}\right|=l$ if $l=5$ or $l \geq 8$ and $\left|G_{l}\right|=l+1$ if $l=6$ or 7 . It is not hard to see that for every $l \geq 5$ the length of any cycle of $G_{l}$ passing all the vertices of $X$ is at least $l$.

So, it remains to prove $n_{5}(l)>l$ for $l=6,7$. Let $l=6$ and suppose that there exists a polyhedral graph $G$ of order 6 with $V(G)=X \cup\{a\}$ such that every cycle which contains the vertices of $X$ is a hamiltonian one. Let $\mathcal{C}(G)$ denote the set of all cycles of $G$. Then we may suppose that $x_{1} x_{2} x_{3} x_{4} x_{5} a x_{1} \in \mathcal{C}(G)$. Clearly, $x_{1} x_{5} \notin E(G)$ which implies that $x_{1} x_{3}$ 
or $x_{1} x_{4} \in E(G)$. If $x_{1} x_{3} \in E(G)$ then $x_{2} x_{5} \notin E(G)$ because otherwise $x_{1} x_{2} x_{5} x_{4} x_{3} x_{1} \in \mathcal{C}(G)$. Thus, $x_{3} x_{5} \in E(G)$ and also $x_{1} x_{4}, x_{2} x_{4} \notin E(G)$ because otherwise $x_{1} x_{2} x_{3} x_{5} x_{4} x_{1}$ or $x_{1} x_{2} x_{4} x_{5} x_{3} x_{1} \in \mathcal{C}(G)$, respectively. Thereby, $x_{2}$ and $x_{4}$ are connected with $a$ which yields that $\left\{x_{3}, a\right\}$ is a cutset, a contradiction. So, we have that $x_{1} x_{3} \notin E(G)$ and $x_{1} x_{4} \in E(G)$ which implies that $x_{3} x_{5} \notin E(G)$ because otherwise $x_{1} x_{2} x_{3} x_{5} x_{4} x_{1} \in \mathcal{C}(G)$. That implies $x_{2} x_{5} \in E(G)$ and thereby $d_{G}\left(x_{3}\right)=2$, a contradiction.

Now, let $l=7$ and suppose that there exists a polyhedral graph $G$ of order 7 with $V(G)=X \cup\{a, b\}$ such that every cycle which contains the vertices of $X$ is a hamiltonian one. We may assume that $\mathcal{C}(G)$ contains one of the cycles $C_{1}=x_{1} x_{2} x_{3} x_{4} x_{5} a b x_{1}, C_{2}=x_{1} x_{2} x_{3} x_{4} a x_{5} b x_{1}, C_{3}=$ $x_{1} x_{2} x_{3} a x_{4} x_{5} b x_{1}$.

Case 1. $C_{1} \in \mathcal{C}(G)$.

Clearly, $x_{1} x_{5}, x_{1} a, x_{5} b \notin E(G)$. If $x_{1} x_{3} \in E(G)$ then $x_{2} x_{5}, x_{2} a \notin E(G)$ because otherwise $x_{1} x_{2} x_{5} x_{4} x_{3} x_{1}$ or $x_{1} x_{2} a x_{5} x_{4} x_{3} x_{1} \in \mathcal{C}(G)$, respectively. Thus, $x_{3} x_{5} \in E(G)$ which yields $x_{1} x_{4}, x_{2} x_{4}, x_{4} b \notin E(G)$ because otherwise $x_{1} x_{2} x_{3} x_{5} x_{4} x_{1}$ or $x_{1} x_{2} x_{4} x_{5} x_{3} x_{1}$ or $x_{1} x_{2} x_{3} x_{5} x_{4} b x_{1} \in \mathcal{C}(G)$, respectively. That implies $x_{2} b, x_{4} a \in E(G)$ which means that $\left\{x_{3}, a\right\}$ or $\left\{x_{3}, b\right\}$ would be a cutset of $G$, a contradiction. If $x_{1} x_{3} \notin E(G)$ we have $x_{1} x_{4} \in E(G)$ and $x_{3} x_{5}, x_{3} a \notin E(G)$ because otherwise $x_{1} x_{2} x_{3} x_{5} x_{4} x_{1}$ or $x_{1} x_{2} x_{3} a x_{5} x_{4} x_{1} \in$ $\mathcal{C}(G)$, respectively. That implies $x_{2} x_{5} \in E(G)$ which means by planarity that $x_{3} b \notin E(G)$. Thus, $d_{G}\left(x_{3}\right)=2$, a contradiction.

Case 2. $C_{2} \in \mathcal{C}(G)$.

Clearly, $x_{1} x_{5}, x_{4} x_{5} \notin E(G)$. Suppose, first, $x_{1} x_{3} \in E(G)$ then $x_{2} x_{5} \notin$ $E(G)$ because otherwise $x_{1} x_{2} x_{5} a x_{4} x_{3} x_{1} \in \mathcal{C}(G)$. Thereby, $x_{3} x_{5} \in E(G)$ which implies that $x_{1} x_{4}, x_{2} x_{4} \notin E(G)$ because otherwise $x_{1} x_{2} x_{3} x_{5} a x_{4} x_{1}$ or $x_{1} x_{2} x_{4} a x_{5} x_{3} x_{1} \in \mathcal{C}(G)$, respectively. Thus, $x_{4} b \in E(G)$ which yields by planarity $x_{1} a, x_{2} a \notin E(G)$, i.e., $\left\{x_{3}, b\right\}$ would be a cutset of $G$, a contradiction. Suppose, now, $x_{1} x_{3} \notin E(G)$ and $x_{1} x_{4} \in E(G)$. Then $x_{2} x_{5}, x_{3} x_{5} \notin E(G)$ because otherwise $x_{1} x_{4} x_{3} x_{2} x_{5} b x_{1}$ or $x_{1} x_{2} x_{3} x_{5} a x_{4} x_{1} \in \mathcal{C}(G)$, respectively. That yields $d_{G}\left(x_{5}\right)=2$, a contradiction. Suppose $x_{1} x_{3}, x_{1} x_{4} \notin E(G)$ then $x_{1} a \in E(G)$. If, here, $x_{2} x_{5} \in E(G)$ then $x_{3} x_{5} \notin E(G)$ because otherwise $x_{1} x_{2} x_{5} x_{3} x_{4} a x_{1} \in \mathcal{C}(G)$. By planarity, $x_{3} b, x_{4} b \notin E(G)$ which means that $\left\{x_{2}, a\right\}$ would be a cutset of $G$, a contradiction. If $x_{2} x_{5} \notin$ $E(G)$ then $x_{3} x_{5} \in E(G)$ and, consequently, $x_{2} x_{4} \notin E(G)$ because otherwise $x_{1} x_{2} x_{4} x_{3} x_{5} a x_{1} \in \mathcal{C}(G)$. Planarity implies $x_{4} b \notin E(G)$ and, hence, $d_{G}\left(x_{4}\right)=2$, a contradiction. 
Case 3. $C_{3} \in \mathcal{C}(G)$.

Clearly, $x_{1} x_{5}, x_{3} x_{4} \notin E(G)$. Suppose, first, $x_{1} x_{3} \in E(G)$ then $x_{2} x_{4}, x_{2} x_{5} \notin$ $E(G)$ because otherwise $x_{1} x_{3} x_{2} x_{4} x_{5} b x_{1}$ or $x_{1} x_{3} a x_{4} x_{5} x_{2} x_{1} \in \mathcal{C}(G)$, respectively. That implies $x_{1} x_{4}$ or $x_{4} b \in E(G)$. If $x_{1} x_{4} \in E(G)$ then $x_{2} b \notin E(G)$ because otherwise $x_{1} x_{3} x_{2} b x_{5} x_{4} x_{1} \in \mathcal{C}(G)$. Thereby, $x_{2} a \in$ $E(G)$ which implies $x_{3} x_{5}, x_{3} b \notin E(G)$ because otherwise $x_{1} x_{2} x_{3} x_{5} x_{4} x_{1}$ or $x_{1} x_{2} x_{3} b x_{5} x_{4} x_{1} \in \mathcal{C}(G)$, respectively. That gives $d_{G}\left(x_{3}\right)=2$, a contradiction. If $x_{1} x_{4} \notin E(G)$ then $x_{4} b \in E(G)$ which yields $x_{3} x_{5} \notin E(G)$ because otherwise $x_{1} x_{2} x_{3} x_{5} x_{4} b x_{1} \in \mathcal{C}(G)$. Thus, $x_{5} a \in E(G)$ and $\{a, b\}$ would be a cutset of $G$, a contradiction.

Suppose, now, $x_{1} x_{3} \notin E(G)$ and $x_{1} x_{4} \in E(G)$. Then $x_{3} x_{5}, x_{3} b \notin E(G)$ because otherwise $x_{1} x_{2} x_{3} x_{5} x_{4} x_{1}$ or $x_{1} x_{2} x_{3} b x_{5} x_{4} x_{1} \in \mathcal{C}(G)$, respectively. That implies $d_{G}\left(x_{3}\right)=2$, a contradiction.

Suppose, eventually, $x_{1} x_{3}, x_{1} x_{4} \notin E(G)$ then $x_{1} a \in E(G)$. That implies $x_{3} x_{5} \notin E(G)$ because otherwise $x_{1} x_{2} x_{3} x_{5} x_{4} a x_{1} \in \mathcal{C}(G)$. Thereby, $x_{3} b \in$ $E(G)$ and by planarity $x_{2} x_{4}, x_{2} x_{5} \notin E(G)$ which means that $\{a, b\}$ would be a cutset of $G$, a contradiction, and the proof is complete.

\section{References}

[1] B. Bollobás and G. Brightwell, Cycles through specified vertices, Combinatorica 13 (1993) 147-155.

[2] G.A. Dirac, 4-crome Graphen und vollständige 4-Graphen, Math. Nachr. 22 (1960) 51-60.

[3] F. Göring, J. Harant, E. Hexel and Zs. Tuza, On short cycles through prescribed vertices of a graph, Discrete Math. 286 (2004) 67-74.

[4] J. Harant, On paths and cycles through specified vertices, Discrete Math. 286 (2004) 95-98.

[5] R. Diestel, Graph Theory (Springer, Graduate Texts in Mathematics 173, 2000).

[6] A.K. Kelmans and M.V. Lomonosov, When $m$ vertices in a $k$-connected graph cannot be walked round along a simple cycle, Discrete Math. 38 (1982) 317-322.

[7] T. Sakai, Long paths and cycles through specified vertices in $k$-connected graphs, Ars Combin. 58 (2001) 33-65.

Received 3 September 2004

Revised 18 February 2005 